\title{
Synthesis of a single cycle of light with compact erbium-doped fibre technology
}

\author{
Günther Kraussì, Sebastian Lohssi', Tobias Hanke, Alexander Sell, Stefan Eggert, Rupert Huber \\ and Alfred Leitenstorfer*
}

The advent of self-referenced optical frequency combs ${ }^{1,2}$ has sparked the development of novel areas in ultrafast sciences such as attosecond technology 3,4 and the synthesis of arbitrary optical waveforms ${ }^{5,6}$. Few-cycle light pulses are key to these time-domain applications, driving a quest for reliable, stable and cost-efficient mode-locked laser sources with ultrahigh spectral bandwidth. Here, we present a set-up based entirely on compact erbium-doped fibre technology, which produces single cycles of light. The pulse duration of $4.3 \mathrm{fs}$ is close to the shortest possible value for a data bit of information transmitted in the near-infrared regime. These results demonstrate that fundamental limits for optical telecommunications are accessible with existing fibre technology and standard freespace components.

Following the report in 1987 of 6 -fs optical pulses from a dye laser system ${ }^{7}$, the generation of few-cycle transients has been boosted by Ti:Sapphire technology. Using sophisticated intracavity dispersion control, a pulse duration of $4.4 \mathrm{fs}$ has been achieved directly with a resonator ${ }^{8}$. Ti:Sapphire amplifiers operating at reduced repetition rates enable extreme compression in hollow fibres $^{9-11}$. Broadband optical parametric oscillators ${ }^{12}$ and amplifiers ${ }^{13}$ have produced pulses as short as $3.9 \mathrm{fs}$ in the visible ${ }^{14}$ and $8.5 \mathrm{fs}$ in the near-infrared ${ }^{15}$. Very recently, 7.8 -fs pulses at a central wavelength of $1.2 \mu \mathrm{m}$ were implemented with erbium-doped fibre technology ${ }^{16}$. All these results correspond to less than two but more than 1.3 oscillation cycles of the electromagnetic field. To synthesize even shorter pulses, the spectra from femtosecond sources may be shaped in amplitude and phase ${ }^{6}$ or pulse trains at different wavelength may be phase-locked and combined ${ }^{5,17}$. In our experiment, we make use of the inherent stability of fibre laser technology $y^{18,19}$ to construct a single cycle of light through the coherent superposition of two ultrabroadband spectra.

The system is outlined in Fig. 1, showing a mode-locked femtosecond erbium-doped fibre oscillator ${ }^{20}$ operating at a repetition rate of $40 \mathrm{MHz}$, which provides seed pulses for two parallel femtosecond erbium-doped fibre amplifiers (EDFA; ref. 21). In each branch the average power of the femtosecond pulse train is amplified to $330 \mathrm{~mW}$. After coupling into free space, each output beam passes a silicon prism sequence, providing variable dispersion. To generate tailored supercontinua, both pulses are coupled into a standard telecom fibre followed by a splice to a highly nonlinear germanosilicate bulk fibre (HNF; ref. 16). In the HNF, the fundamental pump pulse at $1.55 \mu \mathrm{m}$ is split into two spectral components by means of the interplay of dispersion and self-phase modulation. A solitonic part stabilizes itself by shifting to longer wavelengths. This process provides energy for a dispersive wave, which is pushed towards higher frequencies. The position and bandwidth of these spectral features is determined by the dispersion profile of the HNF and the variable amount of pre-chirp introduced with the silicon prism sequence ${ }^{16,22}$. In branch 1 , we use a nonlinear fibre (HNF1) of length $l=4 \mathrm{~mm}$, a zero crossing of the group velocity dispersion at $\lambda_{\mathrm{ZD}}=1,357 \mathrm{~nm}$ and a dispersion slope of $\beta_{3}=$ $0.081 \mathrm{ps}^{3} \mathrm{~km}^{-1}$ to generate a dispersive wave with a large bandwidth centred at a wavelength of $\lambda_{c}=1,125 \mathrm{~nm}$. A different fibre HNF2 $\left(l=10 \mathrm{~mm}, \lambda_{\mathrm{ZD}}=1,431 \mathrm{~nm}, \beta_{3}=0.041 \mathrm{ps}^{3} \mathrm{~km}^{-1}\right)$ is optimized to produce a broadband soliton at $\lambda_{0}=1,770 \mathrm{~nm}$ in the second branch. Both spectra are depicted in Fig. 2a.

The presented two-branch scheme allows the optimization and compression of each component independently: a pair of F2 prisms with an apex distance of $25 \mathrm{~cm}$ is inserted into branch 1 , featuring a zero crossing of the third-order dispersion close to the central wavelength of the dispersive wave. Spectral components beyond $1,450 \mathrm{~nm}$ are blocked with a razorblade in the Fourier domain. The dispersion in branch 2 is controlled by a pair of SF10 prisms with an apex distance of $5 \mathrm{~cm}$. This set-up compensates for the dispersion of a dielectric edge filter, which isolates the soliton by cutting off wavelength components below $1,600 \mathrm{~nm}$.

The timing jitter between the two branches of our system is less than 50 as when integrating from $1 \mathrm{~Hz}$ up to the Nyquist frequency of $20 \mathrm{MHz}$ (ref. 18). This value is two orders of magnitude smaller than the inverse frequency of light at $1.55 \mu \mathrm{m}$. Therefore, coherent superposition of both spectra by means of a dichroic beam combiner (see Fig. 1) may be used to synthesize extremely short pulses. To this end, the minimum pulse duration in each branch is monitored separately through interferometric (collinear) frequency resolved optical gating (IFROG; refs 23,24) based on second-harmonic generation. A free-standing $\mathrm{LiNbO}_{3}$ crystal with a thickness of $11.5 \mu \mathrm{m}$ serves as a second-order nonlinear element with a large acceptance bandwidth. The retrieved spectral amplitudes agree

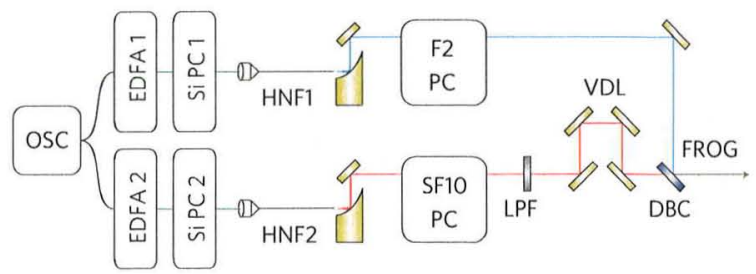

Figure 1 | Set-up of a single-cycle fibre laser system. OSC, femtosecond erbium-doped fibre oscillator; EDFA, erbium-doped fibre amplifier; Si PC, silicon prism compressor; HNF, bulk highly nonlinear fibre for tailored supercontinuum generation; F2/SF10 PC, pulse compressors with F2 and SF10 Brewster prisms; LPF, low-pass filter (cutoff wavelength 1,600 nm); VDL, variable delay line; DBC, dichroic beam combiner. The divergent output leaving each HNF end facet is collimated with off-axis parabolic mirrors. 

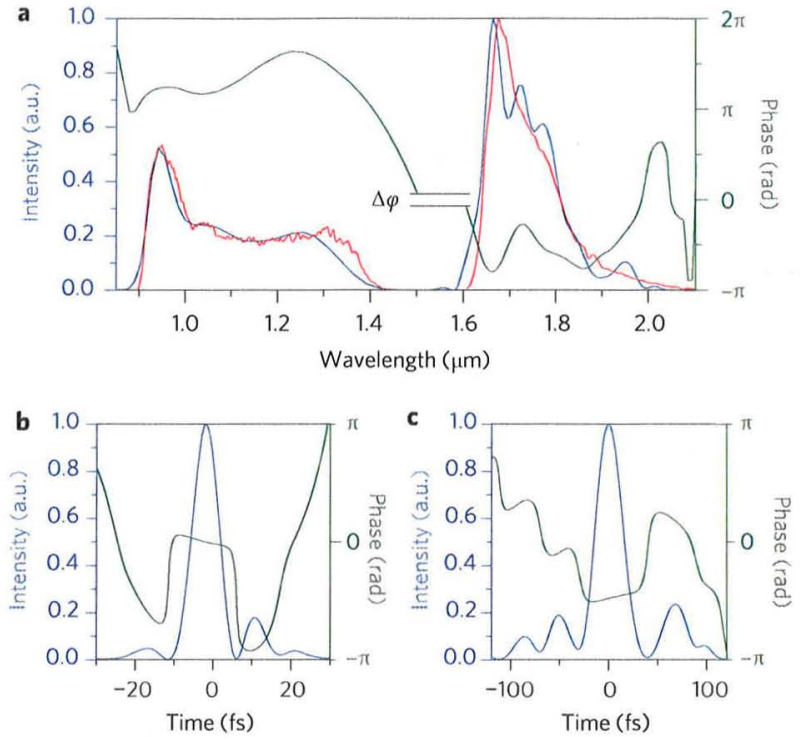

Figure 2 | Spectra and time traces of the pulses generated by the two separate branches. a, Intensity (blue lines) and phase spectra (green) of the dispersive wave in the first branch (left part) and the solitonic pulse from the second branch (right). Intensity spectra measured with a linear spectrometer are depicted as red lines. $\Delta \varphi$, relative phase offset. $\mathbf{b}, \mathbf{c}$, The temporal intensity envelope (blue) and phase (green) feature a pulse duration of $t_{\mathrm{p}}=7.8 \mathrm{fs}$ for the dispersive wave (b) and $t_{\mathrm{p}}=31 \mathrm{fs}$ for the soliton (c).

well with linear measurements and the resulting phase traces are shown in Fig. 2a. The temporal intensity envelope and phase of the dispersive wave are presented in Fig. 2b. The full-width at half-maximum (FWHM) of this pulse is as short as $7.8 \mathrm{fs}$. The temporal intensity and phase for the soliton from HNF2 are depicted in Fig. 2c. Here, the FWHM is equal to $31 \mathrm{fs.}$

We now open up both branches simultaneously and set the relative time delay $\Delta t$ between the pulses with a variable delay stage (see Fig. 1). The position of the retro-reflector is fine-tuned by a piezoelectric transducer. To find the optimum position $\Delta t$ for the synthesis of the shortest pulse, we replace the $\mathrm{LiNbO}_{3}$ crystal in the focus of the IFROG setup and record the nonlinear photocurrent with a GaAs photodiode. Figure 3 shows the interferometric twophoton autocorrelation traces for different values of $\Delta t$. The scanning time $\tau$ of the autocorrelation trace is set by a dispersionbalanced Mach-Zehnder interferometer. At a large pulse separation of $\Delta t=90 \mathrm{fs}$, the time trace consists mainly of two parts: the central region around $\tau=0$ is dominated by a feature similar to the autocorrelation of the more intense dispersive wave from HNF1. In addition, signatures due to the cross-correlation of the dispersive wave and soliton appear around $\tau= \pm 90 \mathrm{fs}$ (indicated by vertical arrows). Surprisingly, these features exhibit an oscillatory structure in $\tau$ with a period of $6 \mathrm{fs}$, corresponding to the inverse central frequency of the soliton. Clearly, no interferometric structure would be expected in a time-averaged linear cross-correlation between two pulses without any spectral overlap. In the nonlinear situation of our experiment, the interference arises because the pulse duration of the dispersive wave is of the same order of magnitude as the oscillation cycle of the soliton, and both components have a well-defined relative phase. As expected, the interferometric cross-correlation signature shifts towards the centre if $\Delta t$ is reduced to $50 \mathrm{fs}$ (see vertical arrows in Fig. 3), although the features around $\tau=0$ remain unchanged. The amplitude of the central maximum at $\tau=0$ starts to depend strongly on $\Delta t$ as soon as temporal overlap occurs for pulse distances below $30 \mathrm{fs}$. This finding is a result of the interference of the central field maximum of the dispersive wave with the individual oscillation cycles of the soliton. It further underlines the excellent degree of mutual coherence between the pulses derived from the two branches of our system. At the optimum relative temporal position between the two components of $\Delta t=0 \mathrm{fs}$, constructive interference arises exactly for the central field maxima of each pulse, whereas the rest of both transients superimposes destructively. This situation is represented by the lowest autocorrelation trace in Fig. 3 where a single oscillation maximum clearly dominates over a pedestal given by the pulse duration of the soliton. Interestingly, the nonlinear autocorrelation changes significantly when we block spectral components beyond $1,800 \mathrm{~nm}$, although the GaAs diode should exhibit a two-photon cutoff at this wavelength. This finding is due to the fact that the leading contribution to the signal is given by a mutual cross-correlation of the entire pulse with the high intensity and photon energy of the central maximum. Note that our system exhibits a slow drift in $\Delta t$ of less than a femtosecond per minute, which is easily stabilized with a feedback loop of a bandwidth of $10 \mathrm{~Hz}$ maximizing the twophoton photocurrent. All data presented in this paper were recorded in the free-running mode.

It turns out that existing schemes for the complete characterization of extremely short optical transients are either too insensitive for our pulse energy of $1 \mathrm{~nJ}$ or not reliable at the large bandwidth extending from $900 \mathrm{~nm}$ to $2.1 \mu \mathrm{m}$. To obtain the total intensity envelope and relative phase, we proceed as follows. The amplitude and phase of dispersive wave and soliton alone have already been determined using IFROG (see Fig. 2), respectively. Because no geometry change occurs with respect to this situation except for the unblocking of both branches at the same time, only three parameters are missing to fully reconstruct the synthesized pulse shape. First, the relative intensity ratio is easily obtained by a linear measurement with a calibrated optical spectrum analyser. Second, a linear phase added to one of the pulses controls the relative temporal delay $\Delta t$ between the two intensity maxima. Third, a

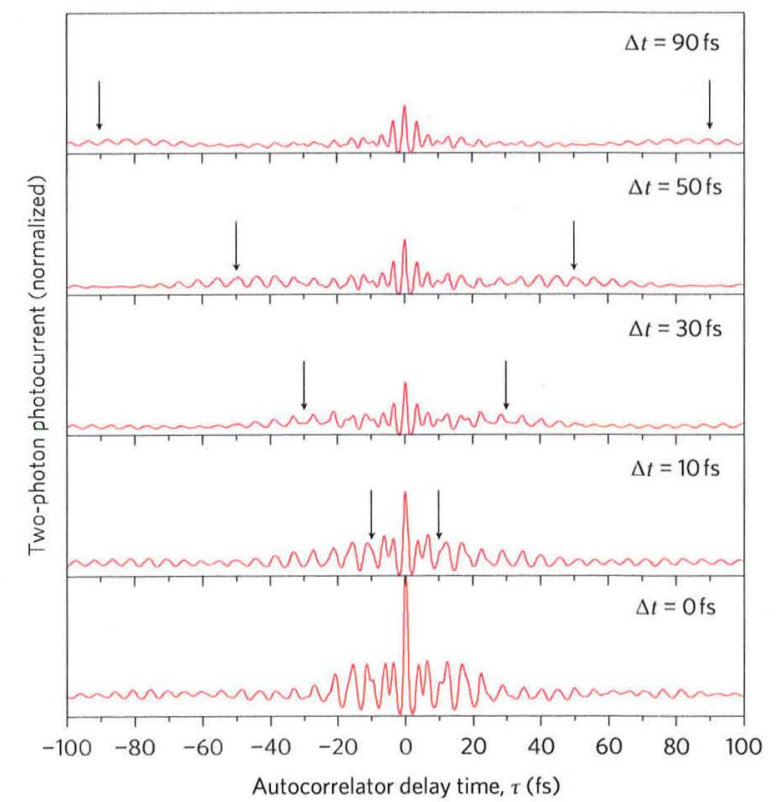

Figure 3 | Fringe-resolved second-order autocorrelations for different time delays $\Delta t$ between dispersive wave and soliton. Red lines: two-photon signal from a GaAs photodiode versus autocorrelator delay time, $\tau$. Vertical arrows: position of nonlinear cross-correlation fringes between both spectral components. At the optimum overlap $(\Delta t=0)$ the signal features an isolated central maximum, indicating the formation of a single-cycle pulse. 

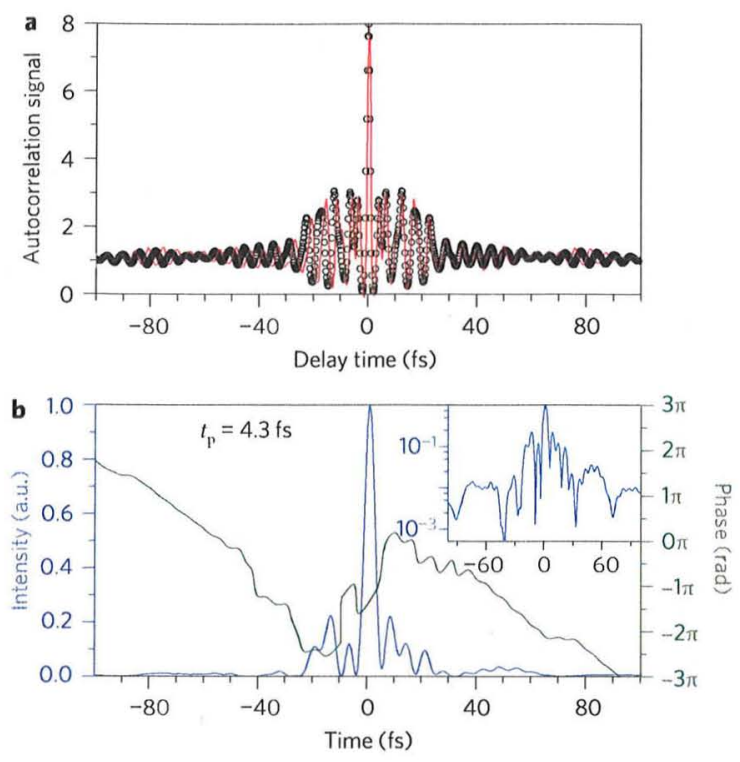

Figure 4 | Characterization of the synthesized single-cycle pulse. a, Second-order autocorrelation trace measured at optimum temporal overlap (red line) and calculated from the combined amplitude and phase spectra in Fig. 2a using a least-square fit varying $\Delta t$ and $\Delta \varphi$ (black circles). b, Temporal intensity envelope (blue lines) and phase (green line) of the combined 4.3-fs light pulse. The inset shows the intensity profile on a logarithmic scale.

constant value $\Delta \varphi$ accounts for the relative phase offset between soliton and dispersive wave. The two parameters linking both phase spectra are now varied and the second-order interferometric autocorrelation corresponding to each situation calculated. A leastsquare fit to the experimental trace in the lowest part of Fig. 3 determines a time delay of $\Delta t=400$ as and a phase offset of $\Delta \varphi=-0.5 \mathrm{rad}$. The fringe-resolved autocorrelation obtained in this way is indicated by the black circles in Fig. $4 \mathrm{a}$, although the red line represents the experiment with the two-photon photodiode. Both traces are in excellent agreement except for some phase shifts in the left part of Fig. 4a, which are caused by a slight deviation from linearity in the fast scanning mode of the Mach-Zehnder interferometer. A Fourier transform of the total amplitude and phase spectra yields the intensity envelope and temporal phase of the synthesized pulse (see Fig. 4b). A FWHM pulse duration of $t_{\mathrm{p}}=4.3 \mathrm{fs}$ is obtained. This value is equal to the oscillation period of light at a wavelength of $1.3 \mu \mathrm{m}$, which is close to the centre of the frequency interval covered by the spectra. The highest intensity of the solitonic pedestal amounts to only $20 \%$ as compared to the central maximum, which contains $50 \%$ of the total pulse energy and exhibits a peak power of $100 \mathrm{~kW}$. Therefore, we conclude that we have generated a singlecycle electromagnetic pulse in the near-infrared. Detailed information on the accuracy of our procedure to determine $t_{\mathrm{p}}, \Delta t$ and $\Delta \varphi$ is given in Fig. 5 and in the Methods.

Our system represents an attractive source for applications in attosecond science and extremely nonlinear optics. For example, we currently perform a direct temporal analysis of single plasmonic optical antennas ${ }^{25}$ featuring few-femtosecond radiation damping. Working at a high repetition rate and with moderate pulse energy is crucial for such studies in ultrafast nanophotonics and quantum optics. In a future extension of our setup, optimum parametric amplification of the dispersive wave and soliton before coherent superposition may be achieved with high-energy pump pulses from passively or actively synchronized Ti:Sapphire ${ }^{26}$ and Nd:YAG (ref. 27) systems.

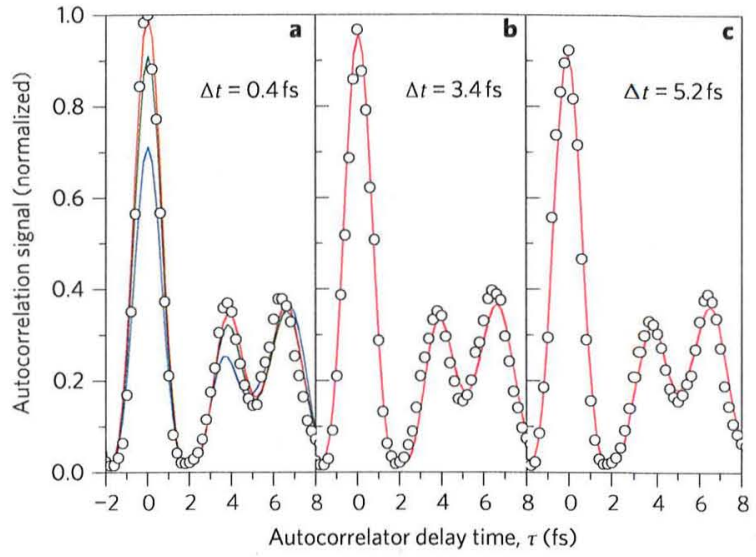

Figure 5 | Sensitivity of the fringe-resolved autocorrelation to $\Delta t$ and $\Delta \boldsymbol{\varphi}$. a, Central parts of the second-order autocorrelation measured at optimum pulse overlap (black circles) compared to simulations assuming a time delay $\Delta t$ of $0.4 \mathrm{fs}$ and phase offsets of $\Delta \varphi=-0.5 \mathrm{rad}$ (red line), $1.1 \mathrm{rad}$ (green line) and 2.6 rad (blue line). b,c, Experimental data and simulations for $\Delta \varphi=-0.5 \mathrm{rad}$, taken at $\Delta t=3.4 \mathrm{fs}$ (b) and $\Delta t=5.2 \mathrm{fs}$ (c), respectively.

\section{Methods}

Two methods are combined to retrieve the full amplitude and phase information of the synthesized pulse. In a first step, we characterize both constituent parts (dispersive wave and soliton) by means of broadband FROG analysis (see Fig. 2). The relative intensity is determined with a calibrated spectrometer. Therefore, only the temporal delay $\Delta t$ between the pulse envelopes and the relative phase $\Delta \varphi$ remain unknown. These two parameters are determined by means of a fringe-resolved nonlinear autocorrelation measurement of the total pulse based on the two-photon photocurrent from a GaAs photodiode. We have carefully chosen this nonlinear element, and a detailed analysis of the two-photon absorption process is crucial: the GaAs detector has a linear absorption cutoff at $900 \mathrm{~nm}$, which is optimally close to the onset of measurable intensity in our spectrum at slightly longer wavelengths (see Fig. 2a). Therefore, no one-photon response needs to be considered when analysing the data in Figs 3 and 4a. On the other hand, this threshold means that the instrument should exhibit a two-photon cutoff at a wavelength of $1,800 \mathrm{~nm}$. Note that our spectrum spans more than one octave and there are spectral components present that extend significantly beyond this point. Nevertheless, when working with the synthesized single-cycle pulses, there is an interesting surprise: the shape of the measured autocorrelation traces changes significantly, even if spectral components of the soliton are blocked between 1,800 and 2,000 $\mathrm{nm}$. Owing to the high intensity in the central cycle of the pulse, the autocorrelation trace may be seen to first order as a mutual cross-correlation of the main peak with the total pulse. Because the central maximum predominantly contains wavelength components far off the two-photon threshold, even the contributions from longer wavelengths are detected with good sensitivity. Our simulations also show that the second-order contributions of the autocorrelation of components between 1,800 and 2,000 nm with themselves are of minor relevance. Therefore, there is no requirement for any model assumptions on the two-photon photoresponse except for the fact that it is of second order. Note that, even without this beneficial effect, our characterization feature would work because, strictly speaking, only the phase relationship between the frequency intervals close to the intensity gap around $1.55 \mu \mathrm{m}$ have to be determined and they are safely within the two-photon absorption bandwidth of the GaAs diode.

We now calculate the two-photon autocorrelation based on the spectral amplitude and phase from Fig. 2a. A least-square fit to the experimental data is performed by varying the missing parameters $\Delta t$ and $\Delta \varphi$. An impression for the accuracy of this procedure is obtained from Fig. 5. The central part of the nonlinear interferogram depends critically on $\Delta \varphi$ (see Fig. 5a), resulting in an experimental error as small as $\pm \pi / 10$. Note that the relative phase is fixed by optimizing both prism compressors for minimum pulse duration. The data fit stringently to a value of $\Delta \varphi=-0.5 \mathrm{rad}$ (see Fig. 5a). The relative amplitude of the interference fringes also changes sensitively with increasing delay time $\Delta t$ (Fig. $5 \mathrm{a}-\mathrm{c}$ ). The excellent amplitude stability ${ }^{16}$ and slow drift of our system allow us to determine a value of $\Delta t=0.4 \mathrm{fs}$ with an accuracy of $\pm 0.7 \mathrm{fs}$. The error margins for $\Delta t$ and $\Delta \varphi$ translate into a contribution of less than 10 as to the uncertainty of the retrieved duration of the single-cycle pulse of $4.3 \mathrm{fs}$. 


\section{References}

1. Jones, D. J. et al. Carrier-envelope phase control of femtosecond mode-locked lasers and direct optical frequency synthesis. Science 288, 635-639 (2000).

2. Udem, T., Holzwarth, R. \& Hänsch, T. W. Optical frequency metrology. Nature 416, 233-237 (2002).

3. Brabec, T. \& Krausz, F, Intense fewncycle laser fields: frontiers of nonlinear optics. Rev. Mod. Phys. 72, 545-591 (2000).

4. Cavalieri, A. L. et al. Intense 1.5-cycle near infrared laser waveforms and their use for the generation of ultra-broadband soft-X-ray harmonic continua. New J. Phys. 9, 242-253 (2007).

5. Shelton, R. K. et al. Phase-coherent optical pulse synthesis from separate femtosecond lasers. Science 293, 1286-1289 (2001).

6. Rausch, S., Binhammer, T., Harth, A., Kärtner, F, X. \& Morgner, U. Few-cycle femtosecond field synthesizer. Opt. Express 16, 17410-17419 (2008).

7. Fork, R. L., Brito Cruz, C. H., Becker, P. C. \& Shank, C. V. Compression of optical pulses to six femtoseconds by using cubic phase compensation. Opt. Lett. $12,483-485$ (1987).

8. Rausch, S. et al. Controlled waveforms on the single-cycle scale from a femtosecond oscillator. Opt. Express 16, 9739-9745 (2008).

9. Sartania, S. et al. Generation of 0.1-TW 5 -fs optical pulses at a $1-\mathrm{kHz}$ repetition rate. Opt. Lett. 22, 1562-1564 (1997).

10. Yamane, $\mathrm{K}$. et al. Optical pulse compression to $3.4 \mathrm{fs}$ in the monocycle region by feedback phase compensation. Opt. Lett. 28, 2258-2260 (2003).

11. Schenkel, B. et al. Generation of 3.8-fs pulses from adaptive compression of a cascaded hollow fiber supercontinuum. Opt. Lett. 28, 1987-1989 (2003).

12. Gale, G. M., Cavallari, M., Driscoll, T. J. \& Hache, F. Sub-20-fs tunable pulses in the visible from an $82-\mathrm{MHz}$ optical parametric oscillator. Opt. Lett. 20 1562-1564 (1995)

13. Wilhelm, T., Piel, I. \& Riedle, E. Sub-20-fs pulses tunable across the visible from a blue-pumped singlepass noncollinear parametric converter. Opt. Lett. 22, 1494-1496 (1997)

14. Baltuška, A., Fuji, T. \& Kobayashi, T. Visible pulse compression to 4 fs by optical parametric amplification and programmable dispersion control. Opt. Lett. 27, 306-308 (2002)

15. Brida, D. et al. Sub-two-cycle light pulses at $1.6 \mu \mathrm{m}$ from an optical parametric amplifier. Opt. Lett. 33, 741-743 (2008)

16. Sell, A., Krauss, G., Scheu, R., Huber, R. \& Leitenstorfer, A. 8-fs pulses from a compact Er:fiber system: quantitative modeling and experimental implementation. Opt. Express 17, $1070-1077$ (2009).
17. Schibli, T. R. et al. Attosecond active synchronization of passively mode-locked lasers by balanced cross correlation. Opt. Lett. 28, 947-949 (2003)

18. Adler, F., Sell, A., Sotier, F., Huber, R. \& Leitenstorfer, A. Attosecond relative timing jitter and 13 fs tunable pulses from a two-branch Er:fiber laser. Opt. Lett. 32, 3504-3506 (2007).

19. Schibli, T. R. et al. Optical frequency comb with submillihertz linewidth and more than $10 \mathrm{~W}$ average power. Nature Photon. 2, 355-359 (2008).

20. Tamura, K., Ippen, E. P., Haus, H. A. \& Nelson, L. E. 77 -fs pulse generation from a stretched-pulse mode-locked all-fiber ring laser. Opt. Lett. 18 , 1080-1082 (1993).

21. Tauser, F., Leitenstorfer, A. \& Zinth, W. Amplified femtosecond pulses from an Er:fiber system: nonlinear pulse shortening and self-referencing detection of the carrier-envelope phase evolution. Opt. Express 11, 594-600 (2003).

22. Tauser, F, Adler, F. \& Leitenstorfer, A. Widely tunable sub-30-fs pulses from a compact erbium-doped fiber source. Opt. Lett. 29, 516-518 (2004)

23. Amat-Roldán, I., Cormack, I. G., Loza-Alvarez, P., Gualda, E. J. \& Artigas, D. Ultrashort pulse characterization with SHG collinear-FROG. Opt. Express 12, $1169-1178(2004)$

24. Stibenz, G. \& Steinmeyer, G. Interferometric frequency-resolved optical gating. Opt. Express 13, 2617-2626 (2005).

25. Merlein, J, et al. Nanomechanical control of an optical antenna. Nature Photon. 2, 230-233 (2008).

26. Sell, A., Leitenstorfer, A. \& Huber, R. Phase-locked generation and field-resolved detection of widely tunable terahertz pulses with amplitudes exceeding $100 \mathrm{MV} \mathrm{cm}^{-1}$. Opt. Lett. 33, 2767-2769 (2008).

27. Chalus, O., Bates, P. K., Smolarski, M. \& Biegert, I. Mid-IR shortpulse OPCPA with micro-Joule energy at $100 \mathrm{kHz}$. Opt. Express 17 , 3587-3594 (2009).

\section{Author contributions}

A.L., G.K., S.L., A.S. and R.H. conceived the experiment, and together with T.H. and S.E. carried it out; G.K., S.L., A.L. and A.S. designed and carried out the data analysis; G.K., S.L R.H. and A.L. co-wrote the paper.

\section{Additional information}

The authors declare no competing financial interests. Reprints and permission information is available online at http:/hpg.nature.com/reprintsandpermissions/. Correspondence and requests for materials should be addressed to A.L. 